\title{
Las perspectivas del trabajo en la sociedad 4.0
}

\section{The prospects of work in society 4.0}

Dr. Daniel Francisco Nagao Menezes

Universidade Presbiteriana Mackenzie, Brasil

nagao.menezes@gmail.com

Fecha de recepción: 13 de noviembre de 2019

Fecha de aceptación: 25 marzo de 2020

\section{RESUMEN:}

Este artículo analiza las discusiones que las sociedades han estado desarrollando para definir la aplicación de sus recursos económicos disponibles en inversiones altamente tecnológicas que priorizan la productividad y competitividad de la economía o las que priorizan las inversiones sociales que reducen las disparidades existentes de ingresos y trabajo. Se investigan las características del progreso tecnológico y su impacto en la estructuración del trabajo, las particularidades del nuevo paradigma tecnológico llamado "industria 4.0" y su impacto en los mercados laborales; y, finalmente, las discusiones sobre las formas de desarrollo a través de la llamada tecnología apropiada y tecnología social, como prioridad para mitigar las repercusiones negativas del avance tecnológico en el mercado laboral.

\section{ABSTRACT:}

This article analyzes the debates societies have been having on how to define the implementation of their economic resources at hand in highly technological investments that prioritize either the economy's productivity and competitiveness or social investments aimed at reducing existing income and labor disparities. This paper investigates the characteristics of technological progress and its impact on labor structuring, the particularities of the new technological paradigm called "Industry 4.0" and its effect on labor markets. Finally, forms of development through the so-called Appropriate Technology and Social Technology are discussed as a priority to mitigate the negative repercussions of technological advance on the labor market.

\section{RESUMO:}

Este artigo analisa as características das discussões que as sociedades vêm desenvolvendo sobre a definição da aplicação de seus recursos econômicos disponíveis em investimentos altamente tecnológicos que priorizam a produtividade e a competitividade da economia ou que priorizam os investimentos sociais que reduzem a renda e as disparidades existentes de renda e emprego. São investigadas as características do progresso tecnológico e seu impacto na estruturação do trabalho, as peculiaridades do novo paradigma tecnológico denominado "indústria 4.0" e seu impacto nos mercados de trabalho; e, finalmente, as discussões sobre as formas de desenvolvimento por meio da chamada tecnologia apropriada e tecnologia social, como prioridade para mitigar as repercussões negativas do avanço tecnológico no mercado de trabalho.

\section{RÉSUMÉ:}

Cet article analyse les caractéristiques des dernières discussions des sociétés sur la définition de la mise en œuvre de leurs ressources économiques disponibles en investissements hautement technologiques qui accordent la priorité soit à la productivité et la compétitivité de l'économie ou à des investissements sociaux qui réduisent les disparités actuelles des revenus et du travail. Il s'agit d'une recherche des caractéristiques du progrès technologique et de son impact sur la structuration du travail, les particularités du nouveau paradigme technologique appelé « Industrie 4.0 » et de son effet sur les marchés de l'emploi. Enfin, il y a des discussions sur les formes de développement à travers ce qui est appelé technologie appropriée et technologie sociale, comme priorité pour mitiger les répercussions négatives du progrès technologique sur le marché de l'emploi.

\section{PALABRAS CLAVES}

TECNOLOGÍA, EMPLEO, INDUSTRIA 4.0, TECNOLOGÍA SOCIAL.
KEYWORDS

TECHNOLOGY,

EMPLOYMENT,

NDUSTRY 4.0,

SOCIAL TECHNOLOGY.
PALAVRAS CHAVE

TECNOLOGIA,

EMPREGO,

INDÚSTRIA 4.0,

TECNOLOGIA SOCIAL.
MOTS CLÉS

TECHNOLOGIE,

EMPLOI,

INDUSTRIE 4.0,

TECNOLOGIE SOCIALE 


\section{INTRODUCCIÓN}

Hoy, las economías mundiales buscan estimular la reanudación de los procesos de desarrollo económico después de la crisis financiera internacional de 2008, que dejó como consecuencia el aumento del desempleo y las condiciones laborales informales de bajos ingresos. La reanudación del crecimiento implica una mayor productividad en las economías y una mayor generación de valor agregado a través de la participación de empresas en cadenas de valor internacionales. Con este fin, el avance de la tecnología digitalizada contribuye cada vez más, mientras que los mercados laborales se reorganizan para eliminar los empleos resultantes de los nuevos modelos tecnológicos.

El Foro Mundial de Desarrollo celebrado en Davos en 2016 fue sobre el tema de la "cuarta revolución industrial”, que para muchos especialistas ya está en marcha, aunque se superpone con la tercera revolución industrial, marcada por la creciente introducción de nuevas tecnologías de información y comunicación. El profesor Klaus Schwab, fundador y organizador del Foro Económico Mundial que se celebra anualmente en Davos, considera que estos cambios son la nueva revolución industrial que dio origen al término "industria 4.0" como una forma alternativa y más avanzada de dinámica industrial anterior. Sin embargo, la preocupación por los efectos negativos del nuevo paradigma en relación con el mercado laboral fue evidente en las discusiones, señalando el aumento en la estructura del desempleo y la informalidad, que afectan particularmente a la fuerza laboral promedio calificada (Foro Económico Mundial, 2016).

En este contexto, la toma de decisiones por parte del Gobierno, las empresas y las personas debe enfrentar el dilema de invertir los recursos disponibles y priorizar su aplicación a tecnologías altamente digitalizadas que aumenten la productividad y reduzcan los costos de producción; y, alternativamente, a tecnologías sociales que apuntan a restablecer empleos y reducir las desigualdades tanto de ingresos como sociales.

Este artículo analiza las características de este dilema que las sociedades, y específicamente las economías en desarrollo, buscan abordar al definir la aplicación de sus recursos tecnológicos y humanos. Particularmente, en los países en desarrollo existen mayores enfrentamientos acerca de priorizar la inversión en recursos disponibles insuficientes: tecnologías más avanzadas o tecnologías que se adaptan socialmente más al perfil de su fuerza laboral. Es el dilema entre buscar una salida a la crisis y reanudar el desarrollo económico mediante la participación en las cadenas de valor internacionales o buscar soluciones para el aumento del desempleo, el aumento de la informalidad en el mercado laboral y la ampliación de las disparidades en el bienestar.

La siguiente sección trata sobre las características del progreso tecnológico y sus impactos en la estructuración del trabajo. A continuación, se presentan las particularidades del nuevo paradigma tecnológico llamado "industria 4.0" y los reflejos en los mercados laborales, como destructor y creador de empleo. La última sección discute las formas de desarrollo a través de la llamada tecnología apropiada y tecnología social, como una forma de resolver el dilema del mejor uso de los recursos de las economías, para mitigar las repercusiones negativas en el mercado de trabajo ante el movimiento del modelo tecnológico.

\section{Progreso tecnológico y estructuración del trabajo}

El progreso tecnológico se logra a través de un conjunto de conocimientos disponibles en una sociedad e incorporados en los trabajadores, aplicados en ciencias y artes industriales, y la inversión de estas capacidades en la producción de bienes y productos. Por lo tanto, los impactos de la evolución tecnológica en el mercado laboral de una economía son directos y se sienten a corto y largo plazo en la división del trabajo, en la generación de valor agregado e ingresos, lo cual, sin embargo, requiere una estructura institucional completa para la calificación y recalificación del trabajador, a fin de permitirle adaptarse a las nuevas condiciones.

La dinámica del desarrollo económico provoca cambios en la estructura productiva de una economía a través del desarrollo de fuerzas productivas y la reasignación sectorial de factores de producción, con la consecuente regionalización del capital y los centros dinámicos de desarrollo. En este contexto, el desarrollo de las fuerzas productivas está impulsado por la introducción de innovaciones tecnológicas, las cuales juegan un papel importante al determinar la reasignación de los factores de producción y la capacidad de acumular. Estas innovaciones actúan en la difusión del crecimiento económico y la eficiencia entre sectores y regiones, de manera diferente y de acuerdo con la capacidad de introducción y asunción del progreso tecnológico por parte de los diversos agentes productivos: mano de obra, productores y Gobierno (Kon, 2016). 
Esta división del trabajo en una sociedad determina la división entre ocupaciones apropiadas para las diferentes ramas productivas, lo cual resulta de adoptar tecnologías también distintas con respecto a la relación capital/trabajo. En las formas de las sociedades de producción capitalistas, el trabajo de cada especialidad productiva se subdivide en operaciones funcionales, a diferencia de las sociedades precapitalistas, cuya división en comercios que diferencian principalmente el papel de los sexos no implica la división del trabajo dentro de los oficios. En paralelo a la división social del trabajo, por lo tanto, la división de fabricación del trabajo es la instalación de los procesos involucrados en la fabricación del producto, en numerosas operaciones realizadas por diferentes trabajadores, de acuerdo con los requisitos de los paradigmas tecnológicos vigentes en cada momento (Braverman, 1977).

El desarrollo de nuevas tecnologías ha sido, a lo largo de la evolución de las sociedades, un agente con gran relevancia que ha llevado a la expansión de oportunidades para combinaciones de recursos materiales y humanos disponibles. El crecimiento constante de la población de las naciones generalmente conduce a la necesidad de aumentar la productividad y la eficiencia de los recursos, lo cual es posible gracias a la innovación tecnológica. Como consecuencia de esto, se observan considerables reflexiones sobre el carácter y la naturaleza del trabajo humano (Tauille, 1987; Moraes Neto, 2003), que se manifiestan a través de una creciente diversidad en las ocupaciones del sistema económico. Estas consecuencias se sentirán en mayor o menor medida, ya sea que las innovaciones tecnológicas involucren un proceso o sistema de producción completo, la creación de un nuevo producto o simplemente la mejora de las etapas del proceso de producción. Serán de mayor o menor impacto si ocurren rápidamente o si requieren un período de tiempo más largo para caracterizarlas.

La literatura muestra que la introducción de innovaciones tecnológicas por parte de productores públicos y privados implica una elección entre diferentes posibilidades tecnológicas. Esto resultará en la absorción de más o menos mano de obra, con diferentes niveles de calificación, lo cual determinará la estructuración de ocupaciones adaptadas a los procesos productivos elegidos. Entre las técnicas conocidas por la sociedad, van a existir aquellas que maximizarán el potencial social en términos de bienestar y, por lo tanto, apropiadas para esa realidad. La técnica más apropiada depende de lo que se esté dispuesto a sacrificar, así como de la prioridad relativa de los objetivos, ya sean sociales o privados. Por otro lado, las microunidades o las empresas privadas eligen su tecnología con vistas a su desarrollo económico, teniendo en cuenta sus propios objetivos, los recursos a su disposición, la naturaleza del mercado en el que operan y su conocimiento de las opciones tecnológicas disponibles (Stewart, 1983; Ehrenberg y Smith, 2002; Moraes Neto, 2003).

\section{Industria 4.0 y los reflejos en el mercado laboral}

Hoy, las economías mundiales experimentan grandes transformaciones industriales, fuertemente marcadas por la integración entre las actividades industriales y de servicios, caracterizadas por un nuevo paradigma de producción de la llamada industria 4.0. Esta tiene grandes repercusiones en las estructuras económicas internas y externas en el orden económico internacional.

Por lo tanto, las industrias inteligentes, donde las máquinas, los sistemas de almacenamiento y los equipos están en red, y donde los elementos inteligentes intercambian información de forma autónoma, son características de todos los conceptos de la industria 4.0. La automatización ha pasado a ser un elemento clave de estas industrias, al permitir un mejor nivel de integración, comunicación ininterrumpida y flexibilidad. Este sistema permite a los operadores simular acciones para probar y optimizar procesos y productos en la fase de diseño, lo cual reduce costos y tiempos de creación. Asimismo, permite integrar en forma horizontal y vertical los sistemas de tecnología de la información que integran una cadena de valor automatizada mediante la digitalización de datos. En este sentido, los múltiples sistemas de información facilitan identificar las fallas en los procesos de la empresa, lo cual conduce a la optimización de la calidad de producción y el ahorro de energía, con resultados de mayor eficiencia en el uso de recursos.

Por lo tanto, estas formas avanzadas de fabricación implican integrar tecnologías físicas y digitales y otras innovaciones en servicios intangibles, así como la integración de todas las etapas de la cadena de valor, desde la fase de desarrollo hasta el uso final de un producto. Es una dinámica que concierne a un contexto más allá del de la automatización en el proceso industrial, ya que consiste en la integración entre la maquinaria y los otros sistemas que operan en esta producción, incluidas las diferentes actividades industriales y de servicio de la cadena de suministro (CNI, 2016). 
Estas nuevas tecnologías se introdujeron cada vez más en las últimas décadas en las sociedades desarrolladas y recientemente se han expandido a los países en desarrollo. En un inicio, se referían a la microelectrónica, la informática, la biotecnología y la tecnología de materiales. Varias encuestas de empresas han determinado otras tecnologías que son principalmente líderes en la industria y que operan bajo esta nueva configuración: Internet de las cosas, Internet de servicios, robótica avanzada, inteligencia artificial, fabricación aditiva, simulaciones, integración de sistemas verticales y horizontales, Big Data, fabricación en la nube, nanotecnología, ciberseguridad, drones inteligentes y realidad aumentada (Loural, 2014; CNI, 2016; Scalabre, 2007).

Para las empresas, los mercados y todo el sistema económico, los impactos significativos de este nuevo modelo de industria implican reducción de costos, eliminación de desechos, ahorro de energía, reducción de errores, mayor seguridad, conservación ambiental, mayor calidad del producto, intensificación y capacidad más rápida para servir a los consumidores, lo cual puede dar como resultado una producción a mayor escala o una mayor personalización de nuevos productos. El nuevo entorno tecnológico, tanto dentro de las empresas como en su relación con el mercado, produce cambios estratégicos en la toma de decisiones, con considerables impactos económicos, ya que genera cadenas de valor agregado en crecimiento y altera la competitividad de las empresas frente al mercado interno y externo.

Las críticas a la automatización avanzada llaman la atención sobre las preocupaciones por la pérdida de empleos, particularmente en los países en desarrollo. En la reunión de los países industrializados más grandes del grupo G20, se discutieron soluciones para casos en los cuales, algunas empresas utilizan robots y, en consecuencia, causan la pérdida de empleos o, pagan salarios más bajos (Snower, 2017).

Las críticas a la automatización avanzada llaman la atención sobre las preocupaciones por la pérdida de empleos, particularmente en los países en desarrollo. En la reunión de los países industrializados más grandes del grupo G20, se discutieron soluciones para casos en los cuales los robots causan la pérdida de empleos o, con salarios más bajos y medios, en tanto el mercado laboral para actividades más complejas se transforma de manera rápida y desventajosa en las perspectivas de ingresos futuros con la introducción de tecnologías digitalizadas (Snower, 2017).

Los efectos negativos de la industria 4.0 en el mercado laboral se están sintiendo a medida que aumenta la automatización y existe la necesidad de que las habilidades evolucionen de un nivel de estructura ocupacional a otro. Por ejemplo, cuando puede tener lugar la sustitución de esfuerzo manual por parte del mecánico, este se mueve hacia el control, con poder de autocorregirse y proporcionar datos sobre los cuales basar decisiones tales como velocidad, selección de temperatura, etc. Por lo tanto, las ocupaciones más mecanizadas reducen las funciones del operador. Sin embargo, existe la necesidad de otras tareas indirectas como la programación (informática), la ingeniería y las matemáticas, que crean nuevos trabajos especializados.

Los robots colaborativos revolucionan la industria y rompen paradigmas al automatizar la línea de ensamblaje y otras fases del proceso de producción, tales como manejo de materiales, corte, pegado, soldadura, pintura, aplicación de adhesivos, transporte de materiales, alimentación de máquinas, pruebas y análisis de laboratorio.

Por lo tanto, con respecto a las repercusiones de la innovación tecnológica de la automatización industrial, es posible observar en algunas economías que la pérdida de ocupaciones o empleos en la industria puede compensarse con nuevas formas de ocupaciones, pero el resultado neto de estas ocupaciones (las pérdidas y ganancias del mercado laboral) serán diversas para cada condición específica de las economías, de acuerdo con el perfil de calificación de la fuerza laboral. Este determina las condiciones para la introducción de las tecnologías más avanzadas, así como la capacitación de los trabajadores para aprender a colaborar y coexistir con tecnologías inteligentes. Los resultados de estos cambios en la creación y destrucción de empleo se reflejan en la estructura ocupacional de cada economía, dependiendo del perfil de calificación de su fuerza laboral y las condiciones de implementación de innovaciones por sectores de actividad (Kon, 2016).

La investigación empírica muestra que estos cambios en las ocupaciones, con la creación de nuevas formas de funciones laborales, son profundas, aunque graduales, y son ayudadas por el uso de innovaciones intangibles por parte de los trabajadores. Sin embargo, también hay cambios en las funciones de los trabajos existentes que utilizan herramientas de realidad virtual y realidad aumentada para ayudar a los trabajadores tradicionales a aumentar su productividad y hacer que sus trabajos sean más seguros. Se observan transformaciones económicas y sociales relevantes en todos los niveles operativos en los que se extienden. La tasa de difusión, así como los impactos en la división del trabajo en las naciones menos avanzadas, puede conducir a una mayor informalidad y desempleo (Tauille, 1987; Moraes Neto, 2003; IEDI, 2017). 
Desde el punto de vista de la toma de decisiones, los cambios tecnológicos que resultan en la industria 4.0 provienen de cambios considerables en la tecnología de la organización. La unidad de negocios, en el modelo anterior a estos cambios, tiene la característica de que sus decisiones sean tomadas por la inteligencia de la empresa, que está compuesta por quienes aportan conocimientos especializados y no necesariamente se limitan al grupo restringido de directores, lo cual es obligatorio. La tecnoestructura incluye todo, desde los miembros de la presidencia y la junta directiva, hasta los ocupantes de otros puestos de responsabilidad, como los jefes de divisiones o departamentos, que se encargan de coordinar la información de otros empleados (Galbraith, 1985; Mintzberg, 2003).

Este consejo de administración central puede variar considerablemente en número de componentes, grado de responsabilidad y tareas, dependiendo de la estructura de la empresa y la medida en que la empresa se enfrenta a transformaciones externas que requieren acciones que la estructura de administración existente no puede lograr. Con la eliminación de la burocracia, los cambios en la tecnoestructura ocurren a través de tecnologías que interconectan los procesos de toma de decisiones y autoajustan las fases del proceso productivo y distributivo. Del mismo modo, con respecto a la decisión sobre el crecimiento a largo plazo de la empresa, las organizaciones que pueden lograr una mayor flexibilidad en este proceso de ajuste observan mayores posibilidades de expandir su mercado y un mayor crecimiento. Por lo tanto, las transformaciones resultan en considerables ventajas competitivas para las empresas capaces de asumir el nuevo paradigma (Kon, 2016).

La forma en que la acumulación de capital promueve el desarrollo, ya sea a través de cambios en el proceso de producción y organización, o ya sea por nuevos productos, está a su vez relacionada con la división social del trabajo en un momento dado, así como la distribución del ingreso, que actúa no solo al determinar la demanda y, por lo tanto, el empleo (en una visión keynesiana), sino también por el valor de la fuerza laboral y su posterior división ocupacional. Por ende, los cambios en el proceso aumentan la productividad en las ramas de producción existentes, haciendo que el producto sea más barato, cambiando la relación capital/trabajo al reducirlo en algunas ramas y aumentarlo en otras. De esta dinámica puede surgir una reducción en la cantidad de trabajo requerida para producir la misma cantidad de producto, lo cual reduce el empleo en sectores específicos de la economía. A su vez, la aparición de nuevos productos significa la sustitución de la producción local de bienes previamente importados o no consumidos, lo cual resulta en la expansión de la actividad productiva en su conjunto y el aumento neto del empleo.

En cualquier caso, el desarrollo tecnológico implica cambios en la capacidad productiva de las economías y la participación de diferentes factores de producción. Así, este conocimiento técnico y tecnológico actualizado permite la formación de nuevos capitales, cambios en la organización de las empresas y la calificación de los recursos humanos de la sociedad. La capacidad de esta para beneficiarse de estos cambios proviene de la forma en que pueda ajustarse con respecto a la redistribución de estos factores de producción, de modo que la demanda global se ajuste a la expansión del potencial económico, pero también al perfil de su producción.

En cuanto a las ventajas para las nuevas ocupaciones creadas por la automatización, los puntos de vista en la literatura también son contradictorios. Algunos analistas consideran que los resultados son favorables, porque el trabajo se vuelve menos monótono y rutinario, con niveles más altos de educación y formación, así como una mayor remuneración. Otros enfoques señalan que las consecuencias son desfavorables porque el trabajador promedio será desplazado por el equipo de mayor productividad, pues carece de la educación y la capacidad para realizar estas funciones automatizadas. Esto se debe a que estas transformaciones tienen lugar en poco tiempo y la fuerza laboral existente no puede satisfacer la demanda de una mayor calificación sin capacitaciones intensivas; por lo tanto, algunas empresas reemplazan a corto plazo la mano de obra no calificada con trabajadores ya calificados o capaces de adaptarse rápidamente a nuevas ocupaciones (Kon, 2016).

Sin embargo, también puede ocurrir en ciertas compañías que la automatización reduzca los requisitos de calificación laboral, al solicitar menos habilidad del operador después de ciertos niveles de mecanización. El trabajador promedio podrá realizar diferentes tareas en forma cada vez más rápida y fácil, con el uso de máquinas altamente automatizadas (Moraes Neto, 2003).

En resumen, persisten las dudas sobre el equilibrio neto entre el desempleo tecnológico y el empleo compensatorio que resulta de los cambios en la estructura ocupacional; cambios que pueden ser positivos o negativos, dependiendo de la velocidad del crecimiento de la innovación tecnológica y la dirección sectorial de estas inversiones. Lo que queda en consenso es el potencial altamente desestabilizador que alcanza a la estructura ocupacional como resultado de la introducción del progreso tecnológico. 


\section{Tecnología apropiada e ideas de tecnología social}

La discusión sobre la "elección” de tecnología apropiada para las condiciones específicas de la fuerza laboral está presente en el análisis del proceso de desarrollo económico y la división del trabajo de los países menos avanzados. La idea de la tecnología apropiada se originó a principios del siglo XX, cuando Gandhi abogó por el mantenimiento y la difusión de tecnologías tradicionales en las aldeas indias; alentó la producción de alimentos y fertilizantes naturales destinados a satisfacer las necesidades de los residentes locales en cooperativas familiares y con tecnologías tradicionales compatibles con las condiciones financieras, culturales y demográficas de la India. Este enfoque enfatizó que la tecnología debería desarrollarse a partir de una integración del desarrollo socioeconómico y cultural para satisfacer las demandas locales (Herrera, 1983).

Después del abandono de esta idea por un tiempo, hubo una reanudación en 1961, a través del concepto de tecnología apropiada introducido por el economista Ernest F. Schumacher, quien más tarde publicó los criterios que caracterizan esta tecnología en su libro Small is Beautiful, al criticar la economía que sufría transformaciones y dificultades durante una crisis energética en 1973 y con la intensificación de la globalización. Los patrones de producción y consumo han cambiado en la búsqueda de nuevos modelos que reducirían los costos y aumentarían la eficiencia.

La idea de la tecnología apropiada consiste en la aplicación sistemática de métodos, técnicas, procesos y productos, destinados a resolver los problemas identificados por una comunidad, a fin de proporcionar efectos positivos y evitar los efectos negativos de la innovación tecnológica en la sociedad, la economía, la cultura y el medio ambiente donde se aplicará. Así, este concepto se refiere a la elección de la infraestructura tecnológica de un país, para asociarlo con las necesidades de sus usuarios y el entorno en el que fluye, el cual, dependiendo de los objetivos, características y formas de actuar, modele esta estructuración con funciones específicas y apropiadas a las condiciones de la fuerza laboral comunitaria (Kon, 2016).

Si bien los matices de la tecnología adecuada varían según los campos, las áreas y los tipos de aplicaciones, generalmente se reconoce como determinada y elegida para aplicaciones de pequeña escala, alta intensidad, descentralizadas y controladas localmente. Esta tecnología, por lo tanto, se adapta a la ubicación y a las necesidades del usuario, así como a los distintos grupos culturales y geográficos, que pueden obtener diferentes resultados de acuerdo con su aplicación, pues cada productor adoptará el mecanismo que mejor satisfaga su condición inmediata, al utilizar posibles bases tecnológicas para su condición espacial, social y financiera.

Estas características son el resultado de la búsqueda de soluciones propias diferenciadas para cada contexto comunitario, por lo que tiene sentido la solución de crear empleo en forma rápida y eficiente para una fuerza laboral no altamente calificada. En la actualidad, otros autores proponen esta forma de tecnología con énfasis en la condición de que la naturaleza de la tecnología debe centrarse en los individuos, es decir, priorizar las necesidades de generación y empleo de poblaciones locales o regionales específicas que a corto plazo carecen de la capacidad para el desarrollo rápido de más alto conocimiento y calificación.

Según Goldemberg (1978), el término tecnología apropiada puede definirse como un proceso de elección de formas que logran ciertos efectos sociales y ambientales de una tecnología propuesta antes de que se desarrolle, de acuerdo con las especificidades del perfil de fuerza laboral, como un intento de incorporar elementos beneficiosos en las diversas etapas de su desarrollo y uso. Por lo tanto, para ser considerada apropiada, una tecnología debe tener algunos aspectos sociales y económicos específicos para satisfacer las necesidades de los sectores más pobres de la población, preservar el medio ambiente, ser adecuada en relación con los recursos naturales disponibles y aprovechar las fuentes disponibles en el lugar de su aplicación.

Por lo tanto, las opciones más apropiadas en el contexto están orientadas a satisfacer las necesidades de los estratos menos privilegiados en términos de ingresos y habilidades, y, en este sentido, están dirigidas a la aplicación de métodos simples, de pequeña escala, de bajo costo e intensivos en mano de obra. Las comunidades rurales son generalmente más favorecidas porque los beneficios provienen de la posibilidad de reemplazar técnicas antiguas y obsoletas sin dañar el proceso de producción, para que el productor pueda continuar explotando al máximo el potencial de los recursos locales en materias primas, energía y equipamiento, sin perjuicio de la producción.

La tecnología apropiada no puede considerarse como una tecnología retrasada o que se haya superado con los avances introducidos por el nuevo conocimiento que generan las actividades de I + D. Para los trabajadores que tienen poca infraestructura tecnológica en su sistema productivo, la introducción de esta tecnología. Su simplicidad 
puede mejorar las características de los sistemas productivos que promueven el desarrollo económico y social de esa parte de la fuerza laboral, en condiciones que de otro modo no se alcanzarían a corto plazo. La transferencia de tecnología apropiada permite a estas comunidades hacer crecer, los excedentes de producir y desarrollar de manera sostenible, tomando en cuenta la necesidad de generación de trabajo que requieren menos inversión que la aplicada a las tecnologías más intensivas en capital (Novaes y Dias, 2009).

La transferencia de tecnología apropiada, permite a estas comunidades, incrementar los excedentes de producción de manera sostenible. Lo anterior permite generar empleo con menor inversión (Novaes y Dias, 2009).

Junto con la tecnología apropiada, el concepto de tecnología social tiene sus propias características, pero también es complementario del primero y generalmente se define en vista de la negación de la tecnología convencional centrada en los aspectos de alta intensidad del capital, estimulando el logro del desarrollo solo para capas específicas de la sociedad. Dagnino (2009) muestra que la tecnología convencional es, por su naturaleza, ahorro de mano de obra, a través del reemplazo constante del trabajo humano por trabajo muerto. Esta tecnología está fuertemente segmentada, lo cual no permite que el productor directo ejerza control sobre la producción y, por lo tanto, es alienante, porque suprime la creatividad del productor directo. Además, es jerárquica, ya que requiere la posesión de los medios de producción y el control sobre el trabajo para ser privado. Su objetivo principal es maximizar la productividad para acumular capital, incluso si tiene efectos negativos sobre el empleo.

En los países en desarrollo, donde la investigación y la innovación no son avanzadas, la tecnología convencional se transmite desde las empresas de los países avanzados a través de la importación y se absorbe sin las críticas adecuadas, con el fin de la competitividad internacional. Por lo tanto, la tecnología convencional impone a estos países los estándares requeridos por los mercados de los países de más altos ingresos y también para una cierta capa privilegiada de países en desarrollo. Sin embargo, en estos países en desarrollo, donde a menudo la mayor parte de la fuerza laboral aún no está lista para asumir ciertos trabajos altamente calificados, se ha encontrado que la solución de desarrollo de tecnologías sociales y apropiadas para absorber esta fuerza laboral menos calificada reduce las asimetrías o disparidades en las condiciones de trabajo y remuneración.

La tecnología social se define a partir de un conjunto de características distintas que tienen como objetivo los efectos en el trabajo, en una escala de producción óptima para esas circunstancias, y que son apropiadas para los efectos sobre el medio ambiente, las características de los insumos utilizados en la producción, la tasa de producción posible y el tipo de control ejercido sobre los trabajadores, lo cual la distingue de la tecnología convencionalmente enfocada en la competitividad interna e internacional (Dagnino, 2009). Por lo tanto, esta tecnología reúne características que permiten incluir a los pequeños productores y consumidores de bajo poder económico de una manera más protegida y específica para satisfacer las necesidades de esta fuerza laboral menos calificada. En este sentido, el incentivo específico se dirige al potencial y la creatividad del productor directo y los usuarios, a través de modelos capaces de habilitar económicamente a empresas como cooperativas populares y pequeños emprendimientos, con una alta relación mano de obra/capital (Novaes y Dias, 2009).

Cabe señalar que la tecnología social también es una tecnología apropiada para generar respuestas más adecuadas a problemas locales específicos y orientarse a la producción colectiva, pero no simple y necesariamente basada en el mercado. En la década de 1970, se discutió mucho sobre la tecnología adecuada y en la década de 1980 perdió importancia y fue objeto de críticas. Sin embargo, las crisis internacionales más recientes, incluida la crisis financiera de 2007-2008, han provocado un alto desempleo incluso en los países desarrollados. Esta situación ha llevado a la reanudación de las discusiones sobre las formas de resolver estos problemas al revisar algunos de los conceptos de tecnología apropiada e incorporar otras contribuciones de estudios más recientes sobre tecnología y su relación con la sociedad. Por lo tanto, se construyó un marco analítico-conceptual para permitir la búsqueda de generación de la tecnología social más efectiva operacionalmente (Dagnino, 2009; Novaes, 2007).

Otros autores explican cómo se extrajeron de la teoría de la economía de la innovación las contribuciones a la idea de la tecnología social, proponiendo un enfoque centrado en la interacción entre los actores dentro de un proceso de innovación. La idea era que las personas que podían abandonar los procedimientos técnicos profundamente arraigados y cambiar los procedimientos de diseño (o sociotécnicos) podrían producir tecnología alternativa para cumplir con especificaciones que difieren de las tecnologías convencionales por compartir los valores y metas que impregnan el escenario deseable de mayor equidad (Novaes y Dias, 2009). 
Estos autores señalan críticamente que la posibilidad de transferir la tecnología generada a cualquier usuario no era muy plausible, como propuso la economía de la innovación, ya que la innovación presupone un proceso en el que los actores sociales interactúan para crear el conocimiento que ellos mismos utilizarán en el proceso. Los criterios para esto eran múltiples y la mayoría de las veces el conocimiento era tácito y no codificado, de modo que el uso de ese conocimiento por parte de otros individuos con diferentes culturas en diferentes entornos y con un grado de heterogeneidad evitaría la transferencia del nuevo conocimiento. Por lo tanto, la tecnología no es el resultado de un proceso en el que los productores simplemente ofrecen productos y procesos, sino que es el resultado de una negociación dinámica que a menudo involucra elementos de naturaleza tácita entre los actores sociales involucrados (Novaes y Dias, 2009).

La respuesta a estas críticas establece que en la tecnología social no podría haber soluciones listas y terminadas para ser transferidas, porque cada contexto requiere sus propias soluciones, pero el conocimiento adquirido se transformaría y sería apropiado para esas particularidades. Una contribución relevante de la economía de la innovación es la idea de que una determinada tecnología no se elige porque es la mejor, sino que se vuelve mejor porque se elige (Novaes y Dias, 2009). Dentro de la tecnología social, la elección significa la aceptación de sus ventajas sociales en lugar de sus cualidades técnicas. Por lo tanto, las críticas a la rentabilidad y la productividad no tienen el mismo sentido que en la tecnología convencional, sino que tienen en cuenta las ventajas sobre el escenario antes de la introducción de la tecnología social. Por lo tanto, las tecnologías se desarrollan y se difunden no solo por factores técnicos destinados a la competitividad, sino principalmente por factores sociales, culturales, políticos y económicos, que están estrechamente relacionados con la formulación de políticas públicas apropiadas para la propuesta de tecnología social.

El movimiento de tecnología social ha sido discutido y actualizado, y en algunos contextos ha logrado resultados de acción considerables. Sin embargo, la continua investigación y reflexión sobre la actualización de modelos y conceptos muestra la necesidad de un proceso dinámico de creación de soluciones específicas de acuerdo con las condiciones locales. De manera global, los conceptos de tecnología social, como mejora de la idea de tecnología apropiada, se elaboran con el objetivo de ser el resultado de la acción de un actor social en un proceso de trabajo en el que también actúan otros actores sociales que se relacionan con artefactos tecnológicos, con el objetivo de producción centrada en el contexto específico. La modificación en el producto, por lo tanto, es apropiada por el actor social y es el resultado de la acción del actor en un proceso de trabajo que permite una modificación cualitativa o cuantitativa en el producto generado, que puede ser apropiado según su interés (Dagnino, 2009).

La necesidad de generar conocimiento y coordinarlo colectivamente para crear valores requiere la participación activa de una gama de actores específicos, representados por el Estado, el Gobierno, las comunidades de investigación y los movimientos sociales. La búsqueda de tecnología adecuada o social debería ser una de las premisas básicas relevantes en un proceso de formulación de políticas públicas si la cuestión de la inversión tecnológica en una economía se centra en la modernización y el aumento de la productividad y la competitividad interna. Pero, también se centra en la inclusión social, a través de la generación de empleos e ingresos para sectores de la población de bajos ingresos, para acceder a tecnología más avanzada.

\section{Consideraciones finales}

En el contexto actual, el concepto de tecnología apropiada como una toma de decisiones para inversiones productivas debe revisarse para ser adaptado a las condiciones específicas que cada economía pueda tener para reanudar o continuar su crecimiento y aumentar el bienestar de las sociedades. En este sentido, la tecnología apropiada va más allá de la connotación que se encuentra en la literatura de priorización de inversiones hacia tecnologías sociales que apuntan a aumentar las oportunidades de trabajo para los sectores más pobres de la población.

Se observa que las tecnologías digitales más avanzadas, que ya se han incorporado de manera efectiva no solo en las economías más fuertes, sino también en los países en desarrollo, tienen efectos tanto en la destrucción rutinaria de empleos en el paradigma anterior como en la generación de nuevas tecnologías, padrones de trabajo y trabajos que abordan las nuevas necesidades creadas.

En este sentido, la toma de decisiones sobre la tecnología apropiada para las condiciones específicas de los recursos y el perfil de la fuerza laboral de cada economía actualmente prioriza las inversiones en tecnologías digitalizadas que apuntan a la inserción competitiva en los mercados nacionales e internacionales, y, finalmente, en términos 
de inversiones en tecnología social, a satisfacer la generación de trabajo e ingresos para la fuerza laboral menos calificada, en paralelo con la creación de inversiones en instituciones destinadas a la calificación y recalificación de la fuerza laboral.

Por lo tanto, elegir entre las alternativas discutidas es un falso dilema. La discusión pertinente se enfoca en la mejor manera de distribuir los recursos entre los objetivos descritos, específicamente y de manera apropiada para cada sociedad, con el fin de contrarrestar la mera búsqueda de productividad y competitividad, y buscar la reducción del desempleo y del trabajo precario.

\section{REFERENCIAS}

Braverman, H. (1977) Trabalho e capital monopolista: a degradação do trabalho no século XX. Rio de Janeiro: Zahar.

CNI (2016) Industry 4.0: a new challenge for Brazilian industry, Especial Survey, 17, N. 2.

Dagnino, R. (2009) Tecnologia social: ferramenta para cons $\neg$ truir outra sociedade. Campinas: IG/INICAMP.

Ehrenberg, R. G. \& Smith, R. S. (2002). A moderna economia do tra $\neg$ balho. São Paulo: Makron Books.

Galbraith, J. K. (1985). O Novo Estado Industrial. São Paulo: Abril Cultural.

Goldemberg, J. (1978). Tecnologia apropriada. Rio de Janeiro: Encontros com a Civilização Bra $\neg$ sileira.

Herrera, A. (1983). Transferencia de tecnología y tecnologías apropiadas. Campinas: Uni $\neg$ camp.

IEDI (2017). Indústria 4.0: a quarta revolução industrial e os desafios para a indús $\neg$ tria e para o desenvolvimento brasileiro. São Paulo: IEDI.

Kon, A. (2016). A Economia do Trabalho. Rio de Janeiro: Alta Books.

Loural, C. A. (2014). Um panorama de novas tecnologias e seus impactos na in $\neg$ dústria. Campinas: Unicamp.

Mintzberg, H. (2003). Criando organizações eficazes: estruturas em cinco configurações. São Paulo: Atlas.

Moraes Neto, B. R. (2003). SéculoXX e trabalho industrial. São Paulo: Xamá.

Novaes, H. T. \& Dias, R. (2009). Contribuições ao Marco Analítiᄀco-Conceitual da Tecnologia Social. En Dagnino, R. Tecnologia social: ferramenta para cons-truir outra sociedade. Campinas: IG/INICAMP.

Novaes, H. T. (2007) O Fetiche da Tecnologia - a experiência das Fábricas Recupe $\neg$ radas. São Paulo: Expressão Popular.

Scalabre, O. (2007). Embracing Industry 4.0 - And Rediscovering Growth. Boston Consulting Groups. Recuperado de https://www.bcg.com.

Snower, D. J. (2017). Porque necessitamos de um forte G20. Frankfurt: Deutschland Edition.

Stewart, F. (1983, julio-septiem $\neg$ bre). Macropoliticas para una tecnologia apropriada: intento de classificación. Revista Internacional del Trabajo, 102(3).

Tauille, J.R. \& Oliveira, C.E.M. (1987). Difusão da Automação no Brasil e os Efei $\neg$ tos sobre o Emprego: Uma Resenha da Literatura Nacional, Rio de Janeiro: UFRJ.

Foro Económico Mundial (2016). The Global Risks Report 2016. Ginebra: Foro Económico Mundial. 